\title{
MICROPRÁTICAS DA MÉDIA GERÊNCIA E SUA RELAÇÃO COM CAPACIDADES DINÂMICAS
}

\author{
(iD) Marina de Almeida Cruz ${ }^{1}$, ${ }^{\text {Victor Silva Corrêa }}{ }^{2}$, Daniela Martins Diniz ${ }^{3}$, ${ }^{\text {Damir Lótfi Vaz }}{ }^{4}$ \\ ${ }^{l}$ Doutora em Administração. Pontifícia Universidade Católica de Minas Gerais (PUC Minas).marina.almeida.cruz@gmail.com \\ ${ }^{2}$ Doutor em Administração. Professor do Programa de Pós-Graduação Stricto Sensu em Administração da UNIP. Universidade Paulista (UNIP). \\ victorsilvacorrea@yahoo.com.br \\ ${ }^{3}$ Doutora em Administração. Professora do Programa de Pós-Graduação em Propriedade Intelectual e Transferência de Tecnologia para a \\ Inovação (PROFNIT). Universidade Federal de São João Del Rei (UFSJ).danidiniz09@yahoo.com.br \\ ${ }^{4}$ Doutor em Administração. Professor do Programa de Pós-Graduação Stricto Sensu em Administração da FDC. Fundação Dom Cabral (FDC). \\ samir@fdc.org.br
}

\section{Resumo}

Objetivo: Embora haja crescente interesse de estudos sobre a Média Gerência (MG) no âmbito da estratégia e das organizações, permanecem pouco exploradas pesquisas que analisam a perspectiva das Capacidades Dinâmicas (CD) sob a lente das micropráticas desses agentes. Neste sentido, o objetivo do estudo é identificar e descrever como as micropráticas desempenhadas por profissionais da média gerência de quatro empresas imersas em ambientes competitivos relacionam-se com as dimensões das capacidades dinâmicas.

Metodologia: A estratégia de pesquisa empregada foi qualitativa. O método utilizado foi o estudo de casos múltiplos, com apropriação de entrevistas semiestruturadas realizadas com profissionais da média gerência.

Originalidade: Ao projetar luzes para as funções da MG através das micropráticas, relacionando-as empiricamente às dimensões das CDs, este artigo enfatiza de maneira inovadora a importância e influência da MG no desenvolvimento das três dimensões das capacidades dinâmicas.

Resultados: Os resultados apontam que a média gerência atua de maneira equilibrada na formação, no desenvolvimento e na modificação das três dimensões das capacidades dinâmicas. Uma segunda conclusão é a de que, das cinco funções gerenciais enfatizadas no estudo, a MG parece desempenhar com maior destaque a microprática de sintetizar informações. Contribuições Teóricas: Os resultados contribuem para o avanço da literatura na área de estratégia e de capacidades dinâmicas, particularmente ao chamar atenção para o papel crítico desempenhado pela média gerência ao desenvolvimento das CDs, aspecto predominantemente investigado na literatura sob a perspectiva da alta administração.

Palavras-chave: Média Gerência. Micropráticas. Capacidades Dinâmicas.

\section{MIDDLE MANAGEMENT MICROPRACTICES AND THEIR} RELATIONSHIP WITH DYNAMIC CAPABILITIES

\section{Abstract}

Objective: Although there prevails a growing interest in the studies on middle management (MG) within the scope of strategy and organizations, considerable research that explores the perspective of dynamic capabilities (DC) under the lens of the agents' micropractices remains scarce. Thus, this study identifies and describes the ways in which the micropractices conducted by MG professionals from four companies embedded in the competitive environments are related to the DC dimensions.

Methodology: A qualitative research strategy was employed, and the multiple case study method, with the appropriation of semistructured interviews conducted with MG professionals, was used.

Originality: This study innovatively emphasizes the importance and influence of MG in the development of three DC dimensions (sense, seizing, and reconfiguration) by highlighting the functions of $\mathrm{MG}$ through micropractices, thereby empirically associating it with the DC dimensions. Results: The study results demonstrate that MG acts in the formation, development, and modification of the three DC dimensions in a balanced way. The results also reveal that from the five managerial functions emphasized in this study, MG seems to play a greater role in the micropractices of synthesizing information.

Theoretical contributions: This study contributes to the advancement of literature in the area of strategy and DC, particularly by drawing attention to the critical role played by MG in the DC development, an aspect predominantly investigated in the literature from the senior management perspective.

Keywords: Middle Management, Micropractices, Dynamic Capabilities

\section{MICROPRÁCTICAS DE GESTIÓN MEDIA Y SU RELACIÓN CON CAPACIDADES DINÁMICAS}

\section{Resumen}

Objetivo: Si bien existe un interés creciente en los estudios sobre gestión intermedia (MG) dentro del alcance de la estrategia y las organizaciones, queda poca investigación que explore la perspectiva de las capacidades dinámicas (DC) bajo la lente de las microprácticas de los agentes.

Metodología: La estrategia de investigación empleada fue cualitativa. El método utilizado fue el estudio de casos múltiples, con la apropiación de entrevistas semiestructuradas realizadas con profesionales de nivel medio.

Originalidad: Este documento enfatiza de manera innovadora la importancia y la influencia de la gerencia media en el desarrollo de las tres dimensiones de capacidades dinámicas. Esto se hace enfatizando las funciones de MG a través de microprácticas, relacionándolas empíricamente con las dimensiones DC.

Resultados: Los resultados demuestran que la gerencia media actúa de manera equilibrada en la formación, el desarrollo y la modificación de las tres dimensiones de capacidades dinámicas. Una segunda conclusión es que de las cinco funciones gerenciales enfatizadas en el estudio, la gerencia media parece jugar un papel más importante en la micropráctica de sintetizar información.

Contribuciones teóricas: Los resultados contribuyen al avance de la literatura en el área de la estrategia y las capacidades dinámicas, particularmente al llamar la atención sobre el papel crítico desempeñado por la gerencia media en el desarrollo de los DC, un aspecto predominantemente investigado en la literatura desde la perspectiva de la alta gerencia.

Palabras clave: Gerencia intermedia. Micro prácticas. Capacidades dinâmicas.

Cite as / Como citar

American Psychological Association (APA)

Cruz, M. de A., Correâ, V. S., Diniz, D. M., \& Vaz, S. L. (2020). Micropráticas da média gerência e sua relação com capacidades dinâmicas. Iberoamerican Journal of Strategic Management (IJSM), 19(2), 66-86. https://doi.org/10.5585/riae.v19i2.15167. 


\section{Introdução}

A perspectiva da Média Gerência (MG) tem se destacado nos últimos anos (Pratap \& Saha, 2018). Isto fica evidente pelo crescente interesse que recebe de estudiosos das áreas de estratégia e organizações (Pratap \& Saha, 2018; Tarakci, Ates, Floyd, Ahn, \& Wooldridge, 2018; Gjerde \& Alvesson, 2020). A influência da MG tem sido enfatizada nas literaturas sobre empreendedorismo, criação de conhecimento, mudança organizacional, descentralização administrativa, inovação do modelo de negócios, entre outras (Wooldridge, Schmid \& Floyd, 2008; Ouekouak, Ouedraogo \& Mbengue, 2014; Schaefer \& Guenther, 2016; Groskovs \& Ulhøi, 2019). Tal notoriedade consequentemente faz com que os indivíduos da MG sejam cada vez mais reconhecidos como "agentes centrais para a formulação e implementação de estratégias" (Vaz \& Bulgacov, 2018, p. 382).

Com efeito, Ahearne, Lam e Kraus (2014) demonstram que os indivíduos da MG são fundamentais ao desempenho das empresas, na medida em que são os principais responsáveis pela articulação entre a alta administração e a base da organização, cumprindo simultaneamente funções ascendente e descendente. Tal fato tende a ser ainda mais evidente em ambientes hostis, onde evidenciase hipercompetição (Mirabeau \& Maguire, 2014; Vuori \& Huy, 2016). Tais contextos são caracterizados pela volatilidade, incerteza, complexidade e ambiguidade (Bennett $\&$ Lemoine, 2014), o que limitam a capacidade da alta administração em detectar, interpretar e processar informações (Walsh, 1995). Portanto, tais ambientes competitivos acentuam a necessidade da influência estratégica da MG em sua habilidade de articular recursos que visam a alavancar o desempenho das empresas (Thomas \& Ambrosini, 2015; Guo, Huy, \& Xiao, 2017; Heyden, Fourné, Koene, Werkman, \& Ansari, 2017).

A despeito da relevância da média gerência em contextos hipercompetitivos, a exemplo daqueles visualizados em diferentes setores produtivos no Brasil e no mundo, a influência da MG sobre as dimensões das capacidades dinâmicas de percepção, aproveitamento e reconfiguração, não tem sido objeto de reflexões teórico-empíricas (Leih \& Teece, 2012; Ouekouak et al., 2014), particularmente no âmbito nacional. Por exemplo, em levantamento por títulos de artigos publicados nos últimos cinco anos e disponíveis no Portal de Periódicos da Capes, não foram encontrados trabalhos que buscassem, tal como aqui sugerido, investigar a influência da $\mathrm{MG}$ sobre as CDs e, mesmo no contexto internacional, parece haver carência. A relação entre $\mathrm{MG}$ e CD foi objeto de reflexão até então essencialmente teórica, o que denota a importância de novas pesquisas orientadas para avaliar, empiricamente, a essência de tal fenômeno (Leih \& Teece, 2012). Estabelecer essa relação é de extrema importância, pois, conforme argumentam Tallot e Hilliard (2016, p. 329), "se o desenvolvimento das capacidades dinâmicas não pode ser explicado, então como os gestores podem agir intencionalmente para desenvolvê-las?”.

Tal indagação e as limitações mencionadas na literatura especializada orientam a realização desta pesquisa, cujo objetivo geral é descrever como as micropráticas da média gerência relacionam-se com as dimensões das capacidades dinâmicas. Para realizar este objetivo, o presente artigo apresenta resultados de um estudo qualitativo das micropráticas de nove profissionais da média gerência que atuam 
em quatro empresas da região metropolitana de Belo Horizonte, todas imersas em ambientes competitivos e dinâmicos. Por sua vez, compreender-se-ão micropráticas como atividades individuais abstraídas de seu contexto específico de aplicação e sintetizadas em categorias mais amplas de modo a refletir ações um pouco mais generalizáveis (Felin, Heimeriks, \& Madsen, 2012; Felin, Foss, \& Ployhart, 2015; Radaelli \& Sitton-Kent, 2016).

Sob o conceito de micropráticas, duas noções fundamentais são de nosso interesse particular, a primeira permite compreender o indivíduo como possível unidade de análise (Teece, 2018a, 2018b). Com efeito, este estudo fundamenta-se na "Visão baseada em Atividades" (Burgelman, et al., 2018; Johnson, Melin \& Whittington, 2003), vertente de pesquisa que salienta a necessidade de estudos que abordem práticas e processos relacionados às atividades do dia a dia, e que tenham, ao mesmo tempo, relação com resultados estratégicos. A segunda, por sua vez, relaciona-se à interação micro-meso-macro. Esta pesquisa parte da premissa de que as micropráticas permitem "entender como fatores no nível individual impactam as organizações [...]", e, de maneira análoga, "como relações entre variáveis macro são mediadas por microações e interações" (Felin et al., 2015, p. 576). Neste sentido, ao focalizar no indivíduo, foi possível projetar luzes para considerações de âmbito mais geral, associadas aos níveis meso e macro.

Em relação à estrutura do artigo, apresentamos a seguir as principais bases de sustentação teórica atreladas às literaturas sobre MG e CD. Tais esclarecimentos permitiram-nos, posteriormente, relatar os procedimentos metodológicos adotados na pesquisa, bem como avançar na descrição e análise dos dados coletados. Finalmente, concluiremos com as considerações finais, dando destaque para as contribuições teóricas e práticas derivadas desta pesquisa, bem como as limitações e sugestões para estudos futuros.

\section{Fundamentação teórica}

\section{Micropráticas da média gerência}

Desde a década de 1970, a literatura sobre MG tem ganhado particular destaque no campo da estratégia organizacional (Floyd \& Wooldridge, 2017). Considerada responsável por mediar, negociar e estabelecer conexões entre os níveis operacional e estratégico das empresas (Castañer \& Yu, 2017; Wooldridge \& Floyd, 2017), a MG acaba por exercer significativa influência no próprio desempenho das organizações como um todo (Ahearne et al., 2014). Em relação ao nível operacional, a MG atua principalmente como "agente de mudança", fomentando a adaptação e a implementação de estratégias deliberadas (Huy et al., 2014; Heyden et al., 2017). Já em relação à alta administração, a MG colabora com interpretações de questões emergentes e com proposições de novas ações capazes de influenciar o próprio direcionamento estratégico da firma (Mirabeau \& Maguire, 2014; Tarakci et al., 2018). Sendo assim, enquanto "a alta administração cria uma visão ou sonho", cabe à média gerência a responsabilidade por estabelecer e implementar "[...] conceitos concretos para solucionar e transcender 
as contradições decorrentes das lacunas entre o que existe no momento e o que a alta gestão espera criar." (Nonaka, 1988, p. 9).

Embora diversos papéis e atividades estratégicas já tenham sido associados aos indivíduos da MG (Rouleau et al., 2015; Guo et al., 2017; Shi et al., 2009), existe um conjunto de quatro micropráticas que são recorrentemente atribuídas à $\mathrm{MG}$ em função de sua influência sobre o desempenho organizacional: "Sintetizar Informações", "Defender Alternativas", "Facilitar a Adaptação" e "Implementar a Estratégia Deliberada" (Floyd \& Wooldridge, 1992; 1997; Pappas \& Wooldridge, 2007; Mantere, 2008; Ahearne et al., 2014). Tais micropráticas podem ser classificadas, por um lado, de acordo com a direção da influência estratégica exercida pelos gestores intermediários na hierarquia formal da organização, sendo ascendente (de baixo para cima), ou descendente (de cima para baixo). Por outro lado, as micropráticas também são classificadas segundo a sua relação com o atual status quo e estratégia organizacional, seja de maneira divergente ou integrativa.

Sintetizar Informações (SI) é uma microprática ascendente e integrativa que se refere à capacidade da MG em interpretar, avaliar e combinar informações estratégicas dos ambientes externo e interno de determinada firma, informando-as aos membros da alta administração (Wooldridge et al., 2008). Defender Alternativas (DA) também emerge como atividade ascendente, mas divergente, implicando ao gestor intermediário apresentar aos superiores o potencial de ações distintas daquelas conduzidas pela própria organização (Mantere, 2005; Ahearne et al., 2014; Tarakci et al., 2018). Já a Implementação de Estratégias Deliberadas (IED) relaciona-se ao momento no qual a MG realiza as estratégias esperadas pela alta administração, por meio de constante monitoramento das ações desempenhadas pelos subordinados, representando, assim, uma microprática descendente e integrativa (Mantere, 2008). Finalmente, a quarta função relaciona-se à necessidade da MG facilitar a adaptação (FA), uma microprática descendente, embora divergente, orientada para fomentar e desenvolver projetos e demais atividades experimentais que visam propor, acomodar e incluir ajustes nas estratégias deliberadas (Ahearne et al., 2014; Tarakci et al., 2018). A classificação das micropráticas apresentadas segue sintetizada na Tabela 1 . 
Tabela 1 - Tipologia da influência estratégica da média gerência

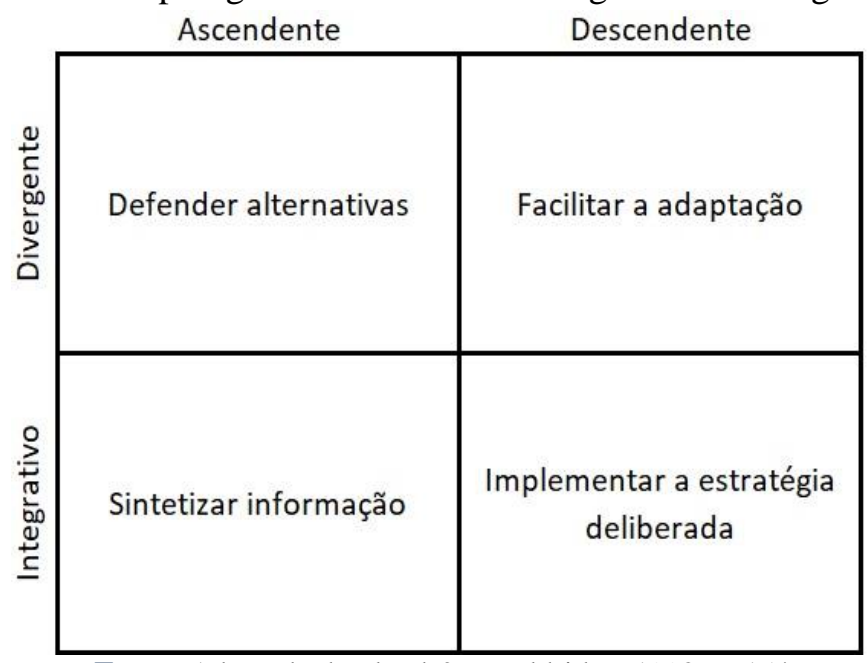

Fonte: Adaptado de Floyd \& Wooldridge, 1992, p. 154.

Não obstante a multiplicidade de consequências derivadas de tais atividades desempenhadas pela MG, a literatura ainda é limitada em explicar a contribuição desse grupo de gestores na "Elaboração de Estratégias" (EE). Isso se torna ainda mais evidente quando se considera que as micropráticas de SI e DA representam apenas uma influência indireta sobre a tomada de decisão formal, esta a cargo da alta administração. Alguns estudos mais recentes começaram a endereçar esta questão, conforme Heyden et al. (2017). Segundo estes autores, a mudança de estratégia terá maior adesão dos funcionários quando tiver origem na MG e contar com o apoio da alta administração (Heyden et al., 2017). Esse protagonismo da média gerência na EE seria fundamental não apenas para melhorar a qualidade das decisões, como também para promover a integração, a coordenação e a comunicação em torno das iniciativas de planejamento (Wolf \& Floyd, 2017). Portanto, uma evidente limitação dos estudos prévios na qual esta pesquisa pretende contribuir reside na melhor compreensão sobre a influência das micropráticas da MG na EE. Apresentamos na próxima seção a relação das micropráticas da média gerência com as capacidades dinâmicas.

\section{Micropráticas e sua relação com as capacidades dinâmicas}

O exame de dimensões das capacidades dinâmicas representa o segundo conjunto de variáveis de interesse desta pesquisa. As CDs são compreendidas como "a habilidade de determinada firma em integrar, construir e reconfigurar competências internas e externas para o direcionamento rápido em ambientes de mudança" (Teece, Pisano \& Shuen, 1997, p. 516), sendo utilizadas pelas empresas para atingir novas formas de vantagem competitiva. A abordagem das CDs dá ênfase ao dinamismo do ambiente como fator capaz de modificar a base de recursos das empresas (Katkalo, Pitelis \& Teece, 2010; Schilke, Hu, Helfat, 2018), considerando a habilidade das empresas em usar, combinar e reconfigurar tais recursos de forma rápida e eficaz (Helfat et. al., 2007; Schilke et al., 2018). Segundo Teece $(2007,2014,2018 b)$, isso é feito à luz da taxonomia de três grupos simultâneos de atividades 
empreendedoras: Percepção (PE), Aproveitamento (AP) e Reconfiguração (RC), os quais envolvem tanto processos e rotinas organizacionais, como decisões gerenciais únicas (Teece, 2016, 2018a).

A percepção é a primeira dimensão das CDs e refere-se à aptidão dos gestores em perceber e avaliar oportunidades e ameaças (Teece, 2007). Isso implica analisar e interpretar continuamente e de forma sistemática informações que se encontram disponíveis no ambiente (Pavlou \& El Sawy, 2011; Teece, 2007; 2018). A segunda etapa, Aproveitamento, visa aproveitar essas oportunidades de mercado e direcionar as ameaças externas, fato que geralmente requer a mobilização de recursos, como investimentos em atividades de desenvolvimento e comercialização (Teece, 2007). Já a terceira dimensão é conceituada como o contínuo alinhamento, realinhamento e reconfiguração de ativos tangíveis e intangíveis, como estruturas, recursos e capacidades (Li \& Liu, 2014; Girod \& Whittington, 2017; Schilke et al., 2018; Teece, 2018). Tais concepções foram sintetizadas por Teece (2007) em modelo analítico apresentado na Figura 1.

Figura 1 - Modelo conceitual das três dimensões das capacidades dinâmicas

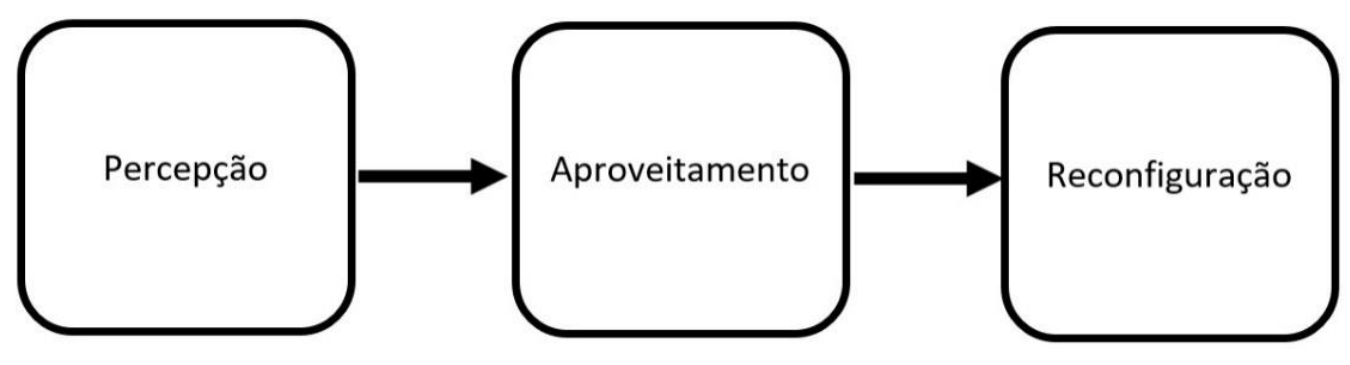

Fonte: Adaptado de Teece, 2007, p. 1342.

Observando que as práticas gerenciais são inerentes às três dimensões de $\mathrm{CD}$, Adner e Helfat (2003) criaram o termo "Capacidades Dinâmicas Gerenciais", o qual também passou a ser recorrentemente utilizado por outros autores (Helfat \& Martin, 2015; Teece, 2014, 2016; Teece, Peteraf $\&$ Leih, 2016). Tal perspectiva assume que a heterogeneidade nos ambientes competitivos decorre da tomada de decisões estratégica das firmas. Estas decisões, por sua vez, dependem das habilidades, dos relacionamentos sociais, bem como das crenças e dos modelos mentais dos gestores (Adner \& Helfat, 2003).

Não obstante o destaque atribuído aos gestores para o desenvolvimento das CD, os estudos prévios concentram-se em avaliar, sobretudo, o papel desempenhado pela alta administração, representada pelos altos escalões das empresas, ao passo que pouco se sabe sobre as contribuições da MG. É possível inferir, à luz das reflexões apresentadas até o momento, a existência de eventual sintonia teórica entre as funções gerenciais da $\mathrm{MG}$ por um lado e as dimensões das CD (Percepção, Aproveitamento e Reconfiguração) por outro. Com efeito, Ouakouak, Ouedraogo, \& Mbengue (2014) lançaram uma fagulha de evidência sobre essa questão, demonstrando que o envolvimento da MG na formulação e implementação de estratégias afeta as capacidades organizacionais, as quais, por sua vez, determinam o desempenho da firma. 
Tal relação, ainda pouco explorada envolvendo as micropráticas da $\mathrm{MG}$ e as $\mathrm{CD}$, representa a principal lacuna teórica sobre a qual este estudo pretende contribuir. As escassas evidências empíricas disponíveis indicam ser mais prudente, neste momento, proceder uma investigação descritiva sobre o assunto antes de avançar por meio de exames explicativos baseados em testes de hipóteses. De qualquer maneira, assumimos a possibilidade de emergir dos dados empíricos a evidência de que sintetizar informações, por exemplo, poderia relacionar-se especialmente à capacidade de percepção. "Implementar a estratégia deliberada", por sua vez, poderia associar-se, por exemplo, à dimensão de aproveitamento. Ou ainda, quem sabe, "defender alternativas" relacionar-se-ia a uma atividade da CD ligada à reconfiguração. Essas são apenas elucubrações que poderão surgir, ou não, a partir de nosso estudo, cujos aspectos metodológicos apresentamos a seguir.

\section{Aspectos metodológicos}

Podemos contextualizar esta pesquisa por meio de quatro principais aspectos metodológicos. Inicialmente, empreendemos aqui uma pesquisa pós-positivista e tal deve-se ao fato de estarmos gerando conhecimento baseado na observação de uma realidade objetiva e aplicada sobre os comportamentos de indivíduos (Creswell, 2010), os quais serão reduzidos a um conjunto de micropráticas associadas a dimensões particulares das CDs. A estratégia de pesquisa empregada foi qualitativa (Creswell, 2010), considerada adequada às situações em que os fenômenos não podem ser estudados fora do contexto no qual ocorrem (Creswell, 2010). Este é justamente o caso das micropráticas e das CDs, ambas de natureza processual (Teece, 2007; Eriksson, 2014). A finalidade do estudo foi descritiva (Ridder, Hoon \& McCandless, 2009; Yin, 2015). Buscou-se descrever, em detalhes, as micropráticas desempenhadas pela MG e suas relações com as dimensões das CDs. Finalmente, o método empregado foi o estudo de casos múltiplos, que, conforme aponta Felin et al. (2015), é adequado à investigação das micropráticas. Com efeito, estudos de caso "são geralmente reconhecidos por [...] permitirem levar a novos desenvolvimentos teóricos" (Felin et al., 2015, p. 613), aqui também objeto de interesse.

Tanto a seleção dos casos (empresas imersas em ambientes dinâmicos) como das unidades de análise (micropráticas realizadas por gestores da média gerência) foram baseadas em critérios teóricos, isto é, na literatura subjacente (Creswell, 2010). Enquanto a primeira baseou-se nas reflexões sobre CD (Teece et al., 1997; Katkalo et al., 2010; Schilke et al., 2018), as unidades de análise foram fundamentadas na abordagem da MG e na "Visão Baseada em Atividades" (Johnson et al., 2003), vertente de pesquisa que salienta a necessidade de estudos que abordem práticas e processos relacionados às atividades do dia a dia e que tenham, ao mesmo tempo, relação com resultados estratégicos. Para efeitos deste trabalho, foram considerados "MG" profissionais de níveis gerenciais situados a um ou a dois níveis hierárquicos abaixo do Presidente/CEO da empresa (Dutton \& Ashford, 1993; Wooldridge et al., 2008; Leih \& Teece, 2012). Incluíram-se, aí, gestores responsáveis por divisões 
ou unidades geograficamente dispersas, gestores funcionais, a exemplo daqueles responsáveis pelos setores de marketing, recursos humanos e financeiro, além de executivos responsáveis por projetos. Uma característica-chave dos profissionais da média gerência, enfatizam Dutton e Ashford (1993, p. 398), é que eles "supervisionam os supervisores e são supervisionados por outros".

Quatro empresas da Região Metropolitana de Belo Horizonte imersas em ambientes competitivos foram selecionadas. Destas, nove gestores da média gerência foram entrevistados. Por razões éticas, os nomes das empresas e dos entrevistados foram modificados. A Empresa A é uma indústria familiar de bens de produção, presente no mercado há 30 anos. Com cerca de 200 funcionários diretos, ela atende a demandas customizadas de equipamentos voltados, sobretudo, ao setor da mineração. A Empresa B é uma indústria familiar de bens de consumo, presente no mercado há mais de 40 anos, om mais de 900 funcionários diretos, além de outros prestadores de serviços terceirizados, fabrica produtos para o lar, comercializados ao consumidor final através de varejistas. A Empresa $\mathrm{C}$ atua no setor de saúde, é de capital fechado, não familiar, e se encontra no mercado há aproximadamente 10 anos. Ela possui cerca de 200 funcionários, destinados a prestar serviços para diagnóstico, tratamento e prevenção de doenças de altas complexidades. Finalmente, a Empresa D possui 25 funcionários e atua na área de engenharia mecânica há 25 anos, prestando serviços para geradoras de energia hidroelétrica no país. Com base no exposto, apresentamos na Tabela 2 síntese com as principais características das empresas e os cargos dos gestores intermediários entrevistados.

Tabela 2 - Características das empresas e cargos dos gestores intermediários entrevistados

\begin{tabular}{|l|c|c|c|c|l|}
\hline & $\begin{array}{c}\text { Setor de } \\
\text { Atuação }\end{array}$ & $\begin{array}{c}\text { Familiar? } \\
\text { (Sim / Não) }\end{array}$ & $\begin{array}{c}\text { Idade Aprox. } \\
\text { (Anos) }\end{array}$ & $\begin{array}{c}\text { Funcionários } \\
\text { Diretos (Aprox.) }\end{array}$ & \multicolumn{1}{c|}{ Cargos dos Entrevistados } \\
\hline Empresa A & $\begin{array}{c}\text { Bens de } \\
\text { Produção }\end{array}$ & Sim & 30 & 200 & $\begin{array}{l}\text { Diretora administrativa e financeira } \\
\text { (Cíntia); Diretor Comercial } \\
\text { (Bernardo); Gerente de } \\
\text { Planejamento Estratégico (Juliano) }\end{array}$ \\
\hline Empresa B & $\begin{array}{c}\text { Bens de } \\
\text { Consumo }\end{array}$ & Sim & 40 & 900 & $\begin{array}{l}\text { Diretor Industrial (João); } \\
\text { Diretora Comercial (Alice) }\end{array}$ \\
\hline Empresa C & Saúde & Não & 10 & 200 & $\begin{array}{l}\text { Superintendente Adjunto (Flávio); } \\
\text { Gerente de Recursos Humanos } \\
\text { (Renata); Gerente de Comunicação e } \\
\text { Marketing (Luciana) }\end{array}$ \\
\hline Empresa D & Energia & Não & 25 & 25 & Gerente de Projetos (Bárbara) \\
\hline
\end{tabular}

Fonte: Elaborada pelos autores.

A coleta de dados se fundamentou na entrevista semiestruturada em profundidade, considerada uma das técnicas mais relevantes em estudos de caso (Voss, Tsikriktsis \& Frohlich, 2002; Adami \& Kiger, 2005; Yin, 2015). O roteiro de entrevistas foi direcionado para captar, de maneira detalhada, descrições de atividades desempenhadas pela MG quanto a: (i) percepção de oportunidades e ameaças, bem como necessidades de investimentos e mudanças internas nas organizações; (ii) participação dos membros da média gerência no processo decisório, como na elaboração e implementação de estratégias, e; (iii) formas de atuação da $\mathrm{MG}$ em processos de reconfiguração e mudanças nas empresas. 
A técnica de observação direta (Bonoma, 1985; Creswell, 2010) foi empreendida de maneira informal, ou seja, sem um roteiro estruturado (Yin, 2015), e teve por objetivo a ampliação de evidências advindas da técnica de entrevistas semiestruturadas. Foi realizado o registro das observações por meio de anotações logo após as entrevistas (Creswell, 2010), durante às visitas in loco nas empresas investigadas, com o intuito de captar gestos, comportamentos e comunicações não verbais dos entrevistados.

As anotações e as transcrições das entrevistas foram tratadas à luz da Análise de Conteúdo (Bardin, 1977; Bauer, 2002), agrupadas em duas categorias fundamentais: Capacidades Dinâmicas e/ou Média Gerência. Cada frase ou parágrafo foi alocado em uma ou ambas as categorias. Elas foram utilizadas para as comparações entre casos. Ao mesmo tempo, à análise dos dados buscou-se empregar a "Generalização Analítica", em que as evidências empíricas foram extrapoladas à literatura subjacente. Para aumentar a validade da pesquisa, foram empregados dois testes de caso: validade do construto e confiabilidade. Ao primeiro, buscou-se o encadeamento de evidências ao longo de todo o processo de pesquisa (Voss et al., 2002; Yin, 2015). Ao segundo, apropriou-se de casos múltiplos, permitindo a lógica da replicação literal (Yin, 2015). Os dados resultantes da pesquisa empírica foram apresentados na próxima seção.

\section{Descrição e análise dos dados}

É possível observar, à luz das evidências empíricas, como os médio-gestores parecem capazes de, durante a execução de suas atividades rotineiras, desenvolver algumas micropráticas (MPs) consideradas fundamentais. Dezenove delas, totalmente inéditas na literatura da área, foram identificadas no campo e nomeadas neste estudo. São elas: i.) Avaliar características, necessidades, tendências, mudanças e preferências dos clientes em relação a produtos e serviços (MP1); ii.) Avaliar o posicionamento competitivo da empresa em relação aos concorrentes (MP2); iii.) Buscar dados sistematizados externamente, como pesquisas de mercado realizadas por empresas especializadas (MP3); iv.) Capturar informações sobre movimentos dos concorrentes, fornecedores, clientes, oportunidades e mudanças que estão acontecendo no mercado (MP4); v.) Compartilhar e discutir informações sobre novas ideias com superiores, pares e subordinados (MP5); vi.) Conquistar legitimidade para novas ideias (MP6); vii.) Conseguir informações sigilosas sobre concorrentes e clientes, por meio de redes de relacionamentos (MP7); viii.) Conversar frequentemente sobre assuntos estratégicos (alta gestão e média gerência) (MP8); ix.) Discutir sobre dificuldades de aproveitamento de oportunidades com superiores, pares e subordinados (MP9); x.) Gerenciar parcerias com stakeholders (MP10); xi.) Identificar necessidades de investimentos para melhorar a capacidade de percepção e de aproveitamento de oportunidades (MP11); xii.) Incentivar a troca de informações internamente para otimizar o aproveitamento (MP12); xiii.) Iniciar mudanças em processos (MP13); xiv.) Montar plano de negócios para análise de novas ideias, oportunidades e resolução de problemas (MP14); xv.) 
Participar ativamente da implementação de mudanças necessárias (MP15); vi.) Realizar pesquisa de mercado diretamente com clientes/revendedores (MP16); xvii.) Sistematizar informações internas para embasar a tomada de decisão (MP17); xviii.) Tomar decisões de forma compartilhada (MP18), e; xix.) Trabalhar ativamente na implementação das estratégias (MP19). Mais ainda, dados de campo projetam luzes para a existência de diferentes associações entre as "MP's" desenvolvidas pelos profissionais às funções da MG, por um lado, e às dimensões das $\mathrm{CD}$, por outro.

A Figura 2, a seguir, expande a natureza desta reflexão, ilustrando as associações identificadas na pesquisa de campo.

Figura 2 - Associação das micropráticas às funções da MG e às dimensões das CDs

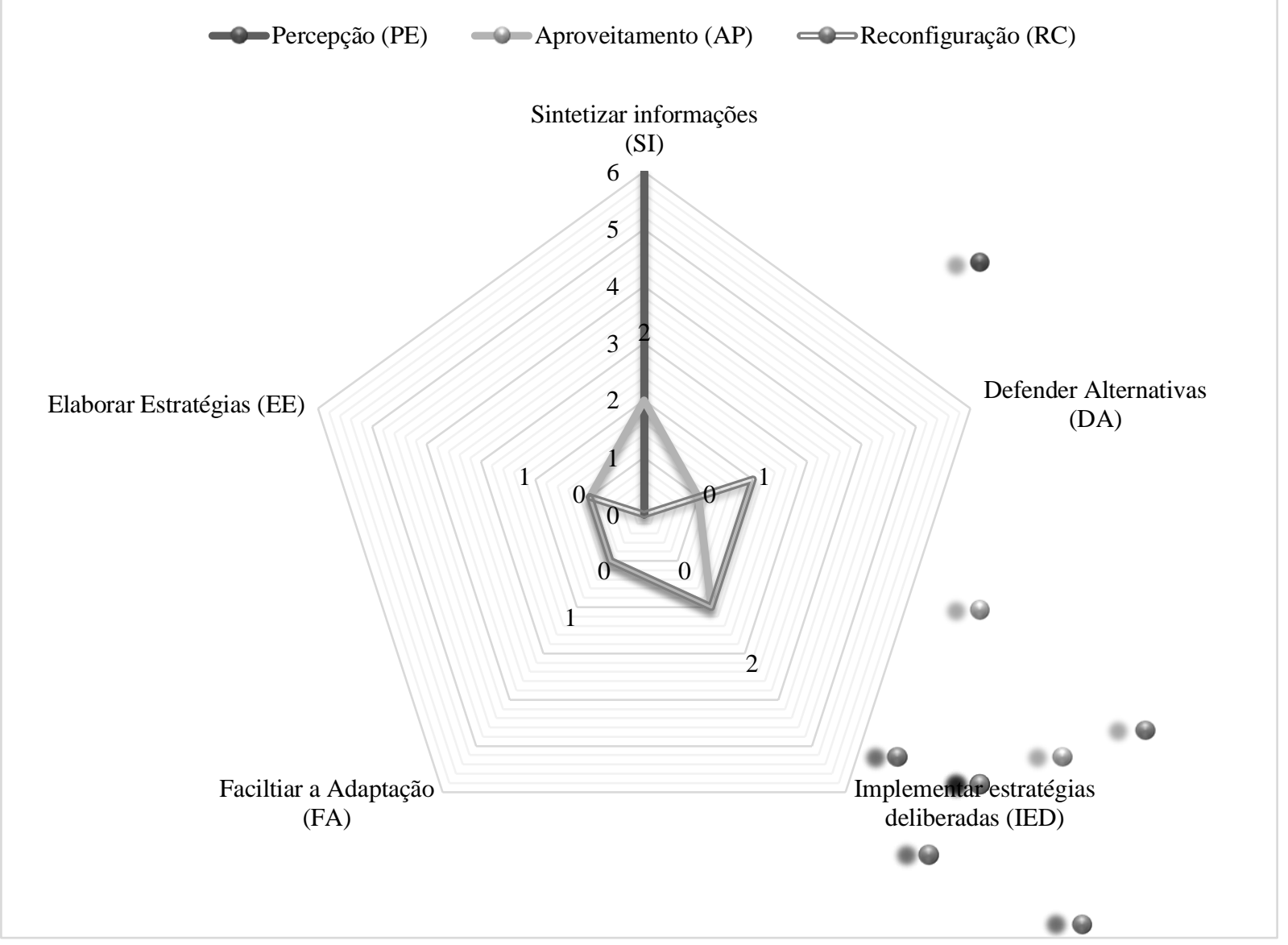

Fonte: Elaborada pelos autores.

É possível observar, à luz da Figura 3, algumas constatações de interesse particular. Das 19 micropráticas identificadas, seis relacionam-se à percepção, sete ao aproveitamento, e outras seis à reconfiguração, denotando relativo equilíbrio das micropráticas às dimensões das CDs. Em relação às funções da MG, contudo, evidências de campo não sugerem uniformidade. De fato, a função de sintetizar informações agrupa oito micropráticas, o dobro do número de MPs identificadas à função de implementar estratégias deliberadas, segundo lugar. As demais funções de defender alternativas, facilitar a adaptação e elaborar estratégias agrupam 3, 2 e 2 micropráticas cada uma, respectivamente. Quando se associa simultaneamente as micrópraticas às funções da MG e às dimensões das CDs, outras 
observações derivam daí. A primeira de que há, em relação ao aproveitamento, micropráticas relacionadas a todas as funções da MG. Em outras palavras, médio-gestores desenvolvem as cinco funções da $\mathrm{MG}$ aqui enfatizadas no aproveitamento das CDs. $\mathrm{O}$ mesmo não aparenta ocorrer com as duas outras dimensões das CDs. Em relação à reconfiguração, gestores parecem exercer apenas quatro funções. Quando associado à percepção, por sua vez, evidências de campo sugerem concentração das micropráticas da MG, apenas, na função de Sintetizar Informações.

Tal é o caso, por exemplo, da "MP1". Dados sugerem que ela se associa à função da MG de "sintetizar informações" e à dimensão de "percepção" das CDs. Salienta Flávio (Empresa C), por exemplo, como a companhia "tem que procurar se adequar". Segundo ele, "não adianta a gente querer competir de igual para igual porque nós não temos esse poder do capital [...]. A gente tem que achar exatamente onde a gente se encaixa nesse mercado, qual é a nossa proposta de posicionamento estratégico para esse mercado". Isso porque, em suas palavras, "se eu for concorrer de igual para igual com grande, fatalmente amanhã vou ser comprado por ele". Note, aí, como Flávio demonstra sintetizar informações do mercado; como percebe, a partir delas, oportunidades nas quais a empresa poderia atuar e aproveitar (Aproveitamento). Categorização análoga ocorre com as "MP's" \#2, \#3, \#4, \#7, \#16. Também a elas parecem estarem associadas a função de sintetizar informações (MG) e a dimensão de percepção $(\mathrm{CD})$.

Em relação à MP2, por exemplo, isso fica claro quando João (Empresa B) salienta: "Nós somos líderes do produto YX tem três anos. Nós não éramos líderes [...], ficamos três meses sem ser líder. Mas, graças a essa inovação constante, a gente tem conseguido resultados melhores”. Veja aí a capacidade do gestor em interpretar de forma sistemática informações do ambiente (Pavlou \& El Sawy, 2011; Teece, 2018). Já a busca sistematizada de dados (MP3) fica evidente, por exemplo, nas pesquisas da Alice (Empresa B). Ela exemplifica: "[quando quero saber] qual é o tamanho do mercado do produto X, eu busco na pesquisa da empresa YZ. Eu sei quais são as marcas líderes, qual é o chefe de cada marca, e assim consecutivamente, inclusive nas categorias que a gente atua". João (Empresa B) complementa: "eu acho que não dá para ter o básico. A gente tem que ter realmente um fluxo de informação dentro da empresa muito grande". Quando relacionado à captura de informações sobre movimentos dos concorrentes (MP4), tal microprática fica saliente, por exemplo, nas ações cotidianas de Bernardo (Empresa A). Ele descreve como os "próprios clientes" são impulsionadores de novas informações. "O cliente fala: 'olha, o fornecedor $\mathrm{C}$ é um fornecedor que tem uma forma de atuar que agrega muito mais valor para mim, $[\ldots]$ do que vocês'. São feedbacks que acontecem nesse dia a dia", diz.

Já “conseguir informações sigilosas sobre concorrentes e clientes por meio de redes de relacionamentos" (MP7) parece possível através da atuação da Bárbara (Empresa D). Ela comenta como informações consideradas fundamentais para a empresa chegam através de suas redes relacionais. "Esse ano a gente já sabe, por exemplo, quais são as tendências: o que vai ser reformado, o que vai ser substituído no mercado no ano que vem.". Segundo ela, "pela rede de informações", isto é, através "das pessoas que nós temos contatos, a gente já consegue prever. Essas pessoas ligam para nós, essas pessoas 
manifestam interesse" (Bárbara/Empresa D). A última microprática associada à percepção das CDs associa-se às atividades de "pesquisa de mercado" realizadas pela MG "diretamente com clientes/revendedores" (MP16). Elas foram identificadas, por exemplo, na atuação da Alice (Empresa B). De acordo com ela, quando a empresa "[...] vai lançar um produto, [...] a gente vai lá [...] conversar com as revendas, para falar: 'olha, qual produto que é mais aceito? Tal ou tal?"” Segundo ela, "todos [da empresa] pedem: 'Alice, olha como é a aceitação de tal produto? Tanto a parte de engenharia, quanto o Marcos Paulo, que é o presidente, todo mundo pede isso" (Alice/Empresa B). Veja como tais micropráticas (\#1, \#2, \#3, \#4, \#7 e \#16) referem-se a uma forma de diagnóstico (Percepção) (Teece, 2007; 2018), ao mesmo tempo em que simbolizam uma capacidade da MG em interpretar, avaliar e combinar informações estratégicas dos ambientes externo e interno, isto é, de sintetizar informações (Mantere, 2008; Wooldridge et al., 2008).

Outras sete MP's (\# 6, \#10,\#12,\#14,\#17,\#18 e \#19), por sua vez, parecem mais associadas à atuação da média gerência em relação ao aproveitamento das CDs. Duas delas (\#14 e \#17) relacionarse-iam especificamente à síntese de informações. A "MP14", em particular, pode ser visualizada na atuação da Cintia (Empresa A). Ela exemplifica: "agora identificamos uma oportunidade de irmos para o estado do Pará". Para aproveitá-la, salienta a gestora, "a gente [montou] o business plan, pegou as informações que a área comercial trouxe e transformou isso em riscos, em oportunidades. Fez uma análise estratégica". Já a "MP17" encontrou ressonância nas atividades da Renata (Empresa C). Ela explica como pesquisas de clima organizacional fornecem diferentes subsídios à tomada de decisão. "A gente mede, a gente tem como saber como que está a satisfação dos profissionais. Aí é levado o resultado dessa pesquisa para os diretores." Segundo ela, a partir das informações, os diretores avaliam aquilo "que eles estão dispostos a investir para a mudança". Outras cinco MPs dividem-se entre as demais quatro funções da MG enfatizadas, sendo: MP6-DA; MP10 e MP19-IED; MP12-FA, e; MP18-EE.

A "conquista de legitimidade para novas ideias" (MP6) pode ser observada, por exemplo, nas atividades do Juliano (Empresa A). Ele ilustra a importância da gerência na atuação desta microprática. "Se não tiver um diretor comprando a ideia, aí não vai". Segundo ele, "muitas ideias que a gente teve pecaram na execução/compartilhamento. Não trazer as pessoas para remar". A microprática de "Gerenciar parcerias com stakeholders", aqui identificada à função de Implementar estratégias deliberadas (IED), demonstra-se presente, por exemplo, na atuação do Bernardo (Empresa A). Ele afirma como uma parceria com uma companhia chinesa está tornado sua empresa "mais elegível nos processos". Segundo ele, "pelo porte da [Empresa A], ela não tem capital social para entrar em projetos de 100 milhões, 200 milhões". Contudo, "a partir do momento que você tem uma figura de consórcio com [os chineses], aí capacita a empresa para entrar em processos desse nível”. Outra microprática também relacionada a IED é a \#19. Salienta Bernardo (Empresa A) como "trabalhar ativamente na implementação das estratégias" é "um desafio [...] todo dia". Em sua percepção, é "claro que [as pessoas] têm resistência à mudança. Mas o nosso trabalho é sacerdotal, é como o Padre José de Anchieta, catequizando. [...] Esse é o trabalho que a gente faz aqui dentro". 
Para "facilitar a adaptação", aproveitando as oportunidades, médio-gestores buscam "incentivar a troca de informações internamente" (MP12). Tal fica evidente, por exemplo, quando Bernardo (Empresa A) fala como a "primeira coisa a gente tem que ter um relacionamento extremamente aberto, em que nós sempre possamos trocar percepções". Para o gestor, "a correspondência tem que ser para os dois lados. [...] Você precisa ter essa capacidade de interação. [...] No dia a dia", defende o gestor, "nós conversamos muito, muito". Isso implica, inclusive, na MP18, associada à função de elaboração de estratégias. Enfatiza Cíntia (Empresa A) como "tomar decisões de forma compartilhada" é algo que, mais recentemente, "está amadurecido" em sua empresa. "A discussão da estratégia dá-se em um fórum coletivo, nas reuniões de diretoria. Muitas vezes, [o CEO] até vem com uma posição e se modifica ao longo da discussão. Então, tem um processo colegiado de tomada de decisão". O mesmo aparenta ocorrer na Empresa C. Segundo Luciana, a "diretoria e a superintendência têm um cuidado de sempre estar envolvendo as lideranças (gerências) nas decisões". Isso porque, ela complementa, "os impactos e [a maneira] como a gente vai conseguir que isso funcione da melhor forma possível vai depender do alinhamento e do envolvimento de toda equipe".

Outras seis micropráticas, por sua vez, aparentam se associar à dimensão da Reconfiguração das CDs. São elas: \#5, \#8, \#9, \#11,\#13 e \#15. Destas, as MPs \#11 e \#13 relacionam-se à função de "Defender Alternativas". Argumenta Juliano (Empresa A), por exemplo, a importância de realizar-se "investimentos para melhorar a percepção e o aproveitamento de oportunidades" (MP11). Ao referir-se sobre a capacidade da empresa em atender aos projetos em curto período de tempo, ele afirmou: algumas "competências a gente tem, [...]mas [quando é relacionado à] área de serviços, por ter prazos mais curtos ainda, eles requerem outras competências [...] que a gente está tendo que trabalhar". Flavio Luiz (Empresa C) fornece outro exemplo. Ele ilustra a importância da modificação do setor de marketing para o aprimoramento da empresa: "a área de marketing [...] está se transformando. [...] A ideia é que a gente tenha um acompanhamento do mercado diariamente. Alguém focado. [...] Hoje a gente faz, mas ainda acho que isso pode melhorar muito", complementa. Já as mudanças de processos (MP13) podem ser observadas também na atuação de Juliano (Empresa A). Responsável por recente processo de mudança estratégica da empresa, ele salienta como "começou a identificar algumas lacunas". A primeira "foi o compartilhamento das informações, a segunda foi tentar trazer os [demais] gerentes para o planejamento estratégico, antes não existia". As MP's \#9 e \#15 relacionam-se à implementação de estratégias deliberadas.

Com efeito, "discutir sobre dificuldades de aproveitamento de oportunidades com superiores, pares e subordinados" demonstra-se fundamental na visão de Juliano (Empresa A). Ao referir-se sobre o tema, ele salientou: a "gente discute muito, onde que é o [problema]. É no custo? É na precificação? É nas competências, é na reconfiguração? Entendeu? [...] Onde é que gente pecou?". Já Luciana (Empresa C) defende a relevância da participação ativa da $\mathrm{MG}$ na implementação de mudanças necessárias (MP15). Ao falar da aquisição de uma empresa realizada pela sua companhia em 2015, ela afirmou como os gestores tiveram que fazer "uma força-tarefa". Em suas palavras, "a gente teve que 
fazer uma divisão de como iria funcionar a parte prática. Tinham pessoas que estavam envolvidas que iriam ter que comunicar aos nossos clientes, aos convênios", entre outras atividades. A penúltima microprática identificada associa-se à função de facilitar a adaptação, refere-se à atividade de "compartilhar e discutir informações sobre novas ideias com superiores, pares e subordinados" (MP5). Ela foi identificada, por exemplo, na atuação de Juliano (Empresa A). Apesar disso, ele reconhece: "é aos pouquinhos. Não é uma coisa que você muda a chavinha assim”. Para ele, “, a gente tem que forçar um pouco para ter essa visão, e aos poucos a gente vai criando uma cultura, realmente, de compartilhamento". Finalmente, a última microprática identificada envolve "conversar frequentemente sobre assuntos estratégicos" (MP8). Tal foi o identificado na atuação da Bárbara (Empresa D). Quando "um sabe de uma oportunidade, ali a gente já senta, a gente já conversa, aí um já tem uma informação. [...]Por isso funciona. Por causa dessa possibilidade de aproximação, e a proximidade que eu falo é proximidade física, a proximidade de sintonia, de conseguir trabalhar em equipe”.

A Tabela 3 , a seguir, reúne as micropráticas identificadas às funções da média gerência, por um lado, e às dimensões das $\mathrm{CD}$, por outro.

Tabela 3 - Micropráticas da Média Gerência, e suas associações às funções da Média Gerência e às dimensões das Capacidades Dinâmicas

\begin{tabular}{|l|c|c|c|}
\hline & Percepção (PE) & Aproveitamento (AP) & Reconfiguração (RC) \\
\hline Sintetizar informações (SI) & $\begin{array}{c}M P 1, M P 2, \\
M P 3, M P 4, \\
M P 7, M P 16 .\end{array}$ & $M P 14, M P 17$ & - \\
\hline Defender Alternativas (DA) & - & $M P 6$ & MP11, MP13 \\
\hline Implementar estratégias deliberadas (IED) & - & $M P 10, M P 19$ & MP9, MP15 \\
\hline Facilitar a Adaptação (FA) & - & $M P 12$ & MP5 \\
\hline Elaborar Estratégias (EE) & - & $M P 18$ & MP8 \\
\hline
\end{tabular}

Fonte: Elaborada pelos autores.

\section{Considerações finais}

O presente trabalho pode fornecer aportes para a literatura sobre capacidades dinâmicas a partir das dimensões conceituais e elementos empíricos delineados por Teece (2007). Contudo, a análise dos dados suscitou algumas reflexões relacionadas ao fenômeno das CDs de forma complementar ao modelo proposto pelo autor. Os resultados possibilitaram observar implicações de micropráticas desempenhadas pela MG para o desenvolvimento das dimensões das capacidades dinâmicas, especialmente em relação ao que emerge da integração entre elas. A análise das micropráticas também permitiu a observação de que praticamente todas elas possuem relação umas às outras, tanto no âmbito de uma mesma dimensão, quanto entre as demais dimensões, e, ainda, entre níveis gerenciais e outros colaboradores da empresa. Desse modo, esta pesquisa corrobora afirmativas de dois trabalhos que se dedicaram a descrever formas de influência da média gerência nas capacidades organizacionais. O primeiro, de Leih \& Teece (2012, p. 21), em que afirmam que "o papel dos gestores é estimular e guiar o desenvolvimento e orquestração 
de capacidades, atividades em que tanto a alta quanto a média gerência devem fazer parte". O segundo, de Ouakouak et al. (2014, p. 313), de que "o envolvimento da média gerência conduz ao desenvolvimento das capacidades organizacionais, o que, por sua vez, resulta em maior performance da firma".

Complementarmente, este artigo permitiu a edificação de importantes conclusões de pesquisa, quatro das quais podem ser destacadas. A primeira, enfatizada anteriormente, é de que a MG aparenta atuar de maneira equilibrada na formação, no desenvolvimento e na modificação das três dimensões das CDs. Das 19 micropráticas mapeadas, 6 relacionam-se à percepção, 7 ao aproveitamento, e outras 6 à reconfiguração. A segunda conclusão de pesquisa é a de que, das cinco funções gerenciais aqui enfatizadas, médios-gestores parecem desempenhar com maior destaque a microprática de sintetizar informações. Com efeito, são 8 micropráticas ao todo, o dobro em relação à função de "implementar estratégias deliberadas", segundo lugar. As três demais funções (DA, FA e EE) compartilham entre si relativa igualdade, com 3, 2 e 2 MPs cada uma, respectivamente. Tal achado é relevante do ponto de vista teórico, pois projeta luzes para os papeis de maior destaque da MG (sintetizar informações e implementar estratégias deliberadas), enfatizando ao mesmo tempo as atividades relacionadas.

A terceira conclusão, por sua vez, está associada às interconexões entre as funções da média gerência e as dimensões das CDs. Dados de campo permitem concluir que os médio-gestores possuem capacidade de empregar as cinco funções gerenciais no aproveitamento das capacidades dinâmicas, denotando a destacada relevância destes profissionais em tal dimensão. $\mathrm{O}$ mesmo não aparenta ocorrer em relação à reconfiguração e à percepção das CDs. Enquanto à primeira são empregadas quatro funções da MG (DA, IED, FA, EE), excetuando aquela relacionada à síntese de informações, na segunda, por sua vez, evidenciam-se micropráticas associadas apenas e justamente à síntese de informações (SI), demonstrando excessiva concentração da média gerência na atuação de somente uma função gerencial. A quarta e última conclusão relaciona-se à anterior. Das quatro micropráticas relacionadas à reconfiguração, aquelas associadas à defesa de alternativas (DA) e à implementação de estratégias deliberadas (IED) agrupam quatro delas, $66 \%$ do total de atividades correspondentes. O mesmo ocorre em relação ao aproveitamento. As funções de sintetizar informações e implementar estratégias deliberadas reúnem 57\% das MPs aí associadas.

\section{Contribuições para teoria e prática}

Ao extrair tais conclusões, este artigo projeta luzes para importantes contribuições teóricas e práticas. A primeira delas relaciona-se à contribuição da média gerência à formação, ao desenvolvimento e à modificação das capacidades dinâmicas. Insere-se sobre a literatura das CDs a noção, ainda hoje prevalecente, de que suas dimensões seriam desenvolvidas, apenas, pela alta gestão. Leih and Teece (2012, p. 28) enfatizam, por exemplo, que na literatura sobre CD, "crescente atenção tem sido dada ao papel desempenhado pela alta gestão". Neste contexto, mesmo quando a MG é 
associada às reflexões sobre CD, estas demonstram-se essencialmente teóricas (Leih \& Teece, 2012). Ao projetar luzes para as funções da MG através das micropráticas e ao relacioná-las empiricamente às dimensões das CDs, este artigo amplia a literatura sobre CD, enfatizando de maneira inovadora a importância e influência da MG no desenvolvimento das CDs por um lado e ampliando com reflexões teórico-empíricas o estudo essencialmente teórico de Leih and Teece (2012) por outro. Deste modo, tal como aqui concluído, as dimensões das CDs parecem ser o resultado das ações da alta gestão, tal como já salientado pela literatura corrente e, também, da média gerência, tal como aqui enfatizado de maneira inovadora. Esta é importante contribuição de pesquisa. Projeta luzes para influência da MG às CDs, ampliando literatura da área, ainda hoje em evolução.

Mas, há algo mais aí, chamando a atenção para outra contribuição de considerável relevância teórica. Implícita à literatura sobre CD, reside noção de que suas dimensões (percepção, aproveitamento, reconfiguração) seriam, de certo modo, sequenciais entre si (Teece, 2007). Em outras palavras, os gestores inicialmente percebem, aproveitam e, somente em seguida, reconfiguram suas capacidades (Teece, 2007). Embora evidências empíricas acenam endossar tal sequenciamento, amplia-o ao mesmo tempo com nova e instigante interpretação: a de que os gestores não precisam necessariamente aproveitar as oportunidades inicialmente para, somente depois, reconfigurarem suas CD. Isso porque, em determinadas situações, os gestores acabam sendo "forçados" a reconfigurar as CDs das empresas para, somente depois, terem condições de aproveitarem dada oportunidade. É o evidenciado, por exemplo, através da MP11 (identificar necessidades de investimentos para melhorar a capacidade de percepção e de aproveitamento de oportunidades).

No âmbito prático, por sua vez, este artigo aparenta duas contribuições principais. A primeira identifica as micropráticas desenvolvidas por profissionais da média gerência. A segunda, ao fazer isto, chama a atenção para a própria necessidade, ainda hoje latente, de incluí-las em programas de formação e capacitação gerenciais, ampliando os conhecimentos, as habilidades e as atitudes dos gestores em relação a tais micropráticas.

\section{Limitações e sugestões para estudos futuros}

Embora forneça importantes subsídios, esta pesquisa apresenta limitações. A primeira refere-se ao desafio dos pesquisadores de avaliar se determinada microprática descrita pelos entrevistados poderia ser considerada, de fato, parte de cada uma das três dimensões das capacidades dinâmicas. Tal ocorrência dificultou, em alguns momentos, a categorização dos dados coletados, bem como a comparação entre os relatos dos gestores das diferentes empresas. Tal situação também foi enfrentada por Jantunen, Ellonen, Johansson (2012), os quais relataram ser esse um dilema comum em estudos qualitativos sobre capacidades organizacionais.

A segunda limitação refere-se ao fato de os dados não terem sido coletados de forma longitudinal, mas somente em um dado momento no tempo. Pesquisas longitudinais, com a coleta de 
dados realizada em diversas etapas com um espaço de tempo maior entre elas, poderiam levar a maior riqueza de detalhes, além de gerar subsídios para identificar mudanças ocorridas na trajetória das organizações ao longo do tempo e os seus impactos nas micropráticas da média gerência. Além disso, os dados foram coletados utilizando especialmente entrevistas semiestruturadas, impossibilitando a triangulação de dados a partir de múltiplas fontes de evidências.

Do exposto, novos estudos teóricos e empíricos demonstram-se necessários. Dentre eles, inserirse-iam, por exemplo: i.) investigar o fenômeno à luz de diferentes técnicas de coleta de dados, triangulando-as; ii.) pesquisar as funções da MG, comparando-as aos níveis contra intuitivos. Por exemplo, seria possível identificar a função de sintetizar informações com níveis descendentes da MG, ampliando tal atributo hoje enfatizado em relação, apenas, aos níveis superiores? iii.) averiguar tais associações à luz de metodologias quantitativas, testando as proposições aqui esboçadas.

Complementarmente, pesquisas futuras poderiam, também, associar outras literaturas ao estudo, em conjunto com as pesquisas sobre capacidades dinâmicas e influência estratégica da média gerência. Como exemplo, é possível citar a literatura de empreendedorismo corporativo, a qual pode fornecer novos elementos para análise de semelhanças, diferenças e possíveis contribuições de tal corrente teórica para analisar as micropráticas desempenhadas por gestores de diferentes níveis hierárquicos, bem como seu impacto no fenômeno das CDs. Outra literatura que pode ser associada é a de redes sociais, a qual pode fornecer subsídios interessantes para a análise de como as redes sociais e organizacionais, especialmente aquelas relacionadas aos gestores de diferentes níveis hierárquicos, podem influenciar o desenvolvimento das CDs.

Finalmente, pesquisas futuras poderiam, também, selecionar amostras de empresas de um mesmo setor, conforme realizado por Jantunen et al. (2012). Tal procedimento amostral pode fornecer elementos de comparação entre as micropráticas realizadas pelos membros da média gerência e também pela alta gestão, e então verificar quão semelhantes ou diferentes são as suas características. Estas e outras questões permanecem, à despeito de suas relevâncias, ainda hoje inexploradas. O campo é fértil. Há possibilidades de diferentes e novas descobertas. Este artigo inseriu-se como estímulo às oportunidades ainda presentes à área.

\section{Referências}

Adami, M. F., \& Kiger, A. (2005). The use of triangulation for completeness purposes. Nurse Researcher, 12(4): 19-29.

Ahearne, M., Lam, S. K., \& Kraus, F. (2014), Performance impact of middle managers' adaptive strategy implementation: The role of social capital. Strategic Management Journal, (35): 68-87.

Bardin, L. (1977), Análise de Conteúdo. Lisboa: Edições 70.

Bauer, M. W., \& Gaskell, G. (2002), Pesquisa Qualitativa com Texto, Imagem e Som: um manual prático. Petrópolis: Vozes. 
Bennett, N., \& Lemoine, G. J. (2014). What a difference a word makes: Understanding threats to performance in a VUCA world. Business Horizons, 57(3): 311-317.

Bogner, W. C., \& Barr, P. S. (2000). Making Sense in Hypercompetitive Environments: A Cognitive Explanation for the Persistence of High Velocity Competition. Organization Science, 11(2): 212226.

Burgelman, R. A., Floyd, S. W., Laamanen, T., Mantere, S., Vaara, E., \& Whittington, R. (2018). Strategy processes and practices: Dialogues and intersections. Strategic Management Journal, 39(3): 531-558.

Castañer, X., \& Yu, H. (2017), The role of middle and top managers in the strategy process. In Floyd, S. W. (Org.) Handbook of Middle Management Strategy Process Research, pp. 13-32, Edward Elgar Publishing.

Creswell, J. W. (2010), Projeto de pesquisa métodos qualitativo, quantitativo e misto. Porto Alegre: Artmed.

Dutton, J. E., \& Ashford, S. J. (1993). Selling issues to top management. Academy of Management, 18(3): 397-422.

Eriksson, T. (2014). Processes, antecedents and outcomes of dynamic capabilities. Scandinavian Journal of Management, 30: 65-82.

Felin, T., Foss, N. J., Heimeriks, K. H., \& Madsen, T. L. (2012). Microfoundations of Routines and Capabilities: Individuals, Processes, and Structure. Journal of Management Studies, 49(8): 13511374.

Felin, T., Foss, N. J., \& Ployhart, R. E. (2015). The microfoundations movement in strategy and organization theory. Academy of Management Annals, 9(1): 575-632.

Floyd, S. W., \& Wooldridge, B. (1992). Middle management involvement in strategy and its association with strategic type: A research note. Strategic Management Journal, 13(S1): 153-167.

Floyd, S. W., \& Wooldridge, B. (1996), The strategic middle manager: How to create and sustain competitive advantage. San Francisco: Jossey-bass Publishers.

Floyd, S. W., \& Wooldridge, B. (1997). Middle management's strategic influence and organizational performance. Journal of Management Studies, 34(3): 465-485.

Floyd, S. W., \& Wooldridge, B. (2017). Handbook of Middle Management Strategy Process Research. London: Edward Elgar Publishing.

Gjerde, S., \& Alvesson, M. (2020). Sandwiched: Exploring role and identity of middle managers in the genuine middle. Human Relations, 73(1): 124-151.

Girod, S. J., \& Whittington, R. (2017). Reconfiguration, restructuring and firm performance: Dynamic capabilities and environmental dynamism. Strategic Management Journal, 38(5): 1121-1133.

Groskovs, S., \& Ulhøi, J. P. (2019). The middle manager in search of business model innovation. Journal of Business Strategy, 40(4): 3-10.

Guo, Y., Huy, Q. N., \& Xiao, Z. (2017). How middle managers manage the political environment to achieve market goals: Insights from China's state-owned enterprises. Strategic Management Journal, 38(3): 676-696. 
Helfat, C. E., Finkelstein, S., Mitchell, W., Peteraf, M. A., Singh, H., Teece, D. J., \& Winter, S. G. (2007), Dynamic capabilities: Understanding strategic change in organizations. London: Blackwell.

Helfat, C. E., \& Peteraf, M. A. (2015). Managerial cognitive capabilities and the microfoundations of dynamic capabilities. Strategic Management Journal, 36(6): 831-850.

Heyden, M. L. M., Fourné, S. P. L., Koene, B. A. S., Werkman, R., \& Ansari, S. (2017). Rethinking 'top-down'and 'bottom-up'roles of top and middle managers in organizational change: Implications for employee support. Journal of management studies, 54(7): 961-985.

Huy, Q. N., Corley, K. G., \& Kraatz, M. S. (2014). From support to mutiny: Shifting legitimacy judgments and emotional reactions impacting the implementation of radical change. Academy of Management Journal, 57(6): 1650-1680.

Jantunen, A., Ellonen, H.-K., \& Johansson, A. (2012). Beyond appearances - Do dynamic capabilities of innovative firms actually differ? European Management Journal, 30(2): 141-155.

Johnson, G., Melin, L., \& Whittington, R. (2003). Micro strategy and strategizing: towards and activity-based view. Journal of Management Studies, 40(1): 3-22.

Katkalo, V. S., Pitelis, C. N., \& Teece, D. J. (2010). Introduction: On the nature and scope of dynamic capabilities. Industrial and Corporate Change, 19(4): 1175-1186.

Leih, S., \& Teece, D. (2012) The Functions of Middle and Top Management in the Dynamic Capabilities Framework. Kindai Management Review, 1: 1-25

Li, D., \& Liu, J. (2014). Dynamic capabilities, environmental dynamism, and competitive advantage: Evidence from China. Journal of Business Research, 67: 2793-2799.

Maciel, C. d. O., Sato, K. H., \& Kato, H. T. (2012). Capacidades dinâmicas e rituais de interação entre alta e média gerência: proposta de um framework. Revista de Administração Pública, 46(2): 599-618.

Mantere, S. (2005). Strategic practices as enablers and disablers of championing activity. Strategic organization, 3(2): 157-184.

Mantere, S. (2008). Role expectations and middle manager strategic agency. Journal of Management Studies, 45(2): 294-316.

Mirabeau, L., \& Maguire, S. (2014). From autonomous strategic behavior to emergent strategy. Strategic management journal, 35(8): 1202-1229.

Nonaka, I. (1988) Toward Middle-up-down Management: Accelerating Information Creation. Sloan Management Review, 29(3): 9-18.

Ouekouak, M. L., Ouedraogo, N., \& Mbengue, A. (2014) The mediating role of organizational capabilities in the relationship between middle managers' involvement and firm performance: an European study. European Management Journal, 32(2): 305-318.

Pappas, J. M., \& Wooldridge, B. (2007). Middle managers' divergent strategic activity: An investigation of multiple measures of network centrality. Journal of management studies, 44(3): 323-341.

Pavlou, P. A.; El Sawy, O. A. (2011). Understanding the elusive black box of dynamic capabilities. Decision sciences, 42(1): 239-273. 
Penrose, E. T. (1959). The Theory of the Growth of the Firm. Oxford: Basil Blackwell, 1959.

Pratap, S., \& Saha, B. (2018). Evolving efficacy of managerial capital, contesting managerial practices, and the process of strategic renewal. Strategic Management Journal, 39(3): 759-793.

Radaelli, G., \& Sitton-Kent, L. (2016). Middle managers and the translation of new ideas in organizations: a review of micro-practices and contingencies. International Journal of Management Reviews, 18(3): 331-332.

Ridder, H., Hoon, C., \& McCandless, A. (2009). The theoretical contribution of case study research to the field of strategy and management. Research Methodology in Strategy and Management, 5(1): 137-175.

Rouleau, L. (2005). Micro-practices of strategic sensemaking and sensegiving: How middle managers interpret and sell change every day. Journal of Management Studies, 42(7): 1413-1441.

Schaefer, T., \& Guenther, T. (2016). Exploring strategic planning outcomes: the influential role of top versus middle management participation. Journal of Management Control, 27(2): 205-249.

Schilke, O., Hu, S., \& Helfat, C. (2018). Quo Vadis, Dynamic Capabilities? A content-analytic review of the current state of knowledge and recommendations for future research. Academy of Management Annals, 12(1): 390-439.

Shi, W., Markoczy, L., \& Dess, G. G. (2009). The role of middle management in the strategy process: Group affiliation, structural holes, and tertius iungens. Journal of Management, 35(6): 1453-1480.

Tallott, M., \& Hilliard, R. (2016). Developing dynamic capabilities for learning and internationalization: A case study of diversification in an SME. Baltic Journal of Management, 11(3): 328-347.

Tarakci, M., Ateş, N. Y., Floyd, S. W., Ahn, Y., \& Wooldridge, B. (2018). Performance feedback and middle managers' divergent strategic behavior: The roles of social comparisons and organizational identification. Strategic management journal, 39(4): 1139-1162.

Thomas, L., \& Ambrosini, V. (2015). Materializing strategy: the role of comprehensiveness and management controls in strategy formation in volatile environments. British Journal of management, 26: 05-S124.

Teece, D. J. (2007). Explicating dynamic capabilities: the nature and microfoundations of (sustainable) enterprise performance. Strategic Management Journal, 28, 1319-1350.

Teece, D. J. (2014). The foundations of enterprise performance: dynamic and ordinary capabilities in an (economic) theory of firms. Academy of Management Perspectives, 28(4): 328-352.

Teece, D. J. (2016). Dynamic capabilities and entrepreneurial management in large organizations: Toward a theory of the (entrepreneurial) firm. European Economic Review, 86: 202-216.

Teece, D. (2018a). Business models and dynamic capabilities. Long Range Planning, 51(1): 40-49.

Teece, D. (2018b). Dynamic capabilities as (workable) management systems theory. Journal of Management \& Organization, 24(3): 1-10.

Teece, D. J., Peteraf, M., \& Leih, S. (2016). Dynamic capabilities and organizational Agility. California Management Review, 58(4): 13-35. 
Teece, D. J., Pisano, G., \& Shuen, A. (1997). Dynamic capabilities and strategic management. Strategic Management Journal, 18(7): 509-533.

Vaz, S. L., \& Bulgacov, S. (2018). Envolvimento Estratégico da Média Gerência: Analisando o Passado e Projetando o Futuro. Revista de Administração Contemporânea, 22(3): 380-402.

Volberda, H. W. (1996). Toward the Flexible Form: How to Remain Vital in Hypercompetitive Environments. Organization Science, 7(4): 359-374.

Voss, C., Tsikriktsis, N., \& Frohlich, M. (2002). Case research in operations management. International Journal of Operations \& Production Management, 22(2): 195-219.

Vuori, T. O., \& Huy, Q. N. (2016). Distributed attention and shared emotions in the innovation process: How Nokia lost the smartphone battle. Administrative Science Quarterly, 61(1): 9-51.

Walsh, J. P. (1995). Managerial and organizational cognition: Notes from a trip down memory lane. Organization science, 6(3): 280-321.

Wolf, C., \& Floyd, S. (2017). Strategic Planning Research: Toward a Theory-Driven Agenda. Journal of Management, 43(6): 1754-1788.

Wooldridge, B., \& Floyd, S. W. (2017). Some middle managers are more influential than others: an approach for identifying strategic influence. In Floyd, S. W. (Org.) Handbook of Middle Management Strategy Process Research, pp. 13-32, Edward Elgar Publishing

Wooldridge, B., Schmid, T., \& Floyd, S. W. (2008). The middle management perspective on strategy process: Contributions, synthesis, and future research. Journal of management, 34(6): 1190-1221.

Yin, R. K. (2015). Estudo de Caso: planejamento e métodos. Porto Alegre: Bookman. 